\title{
Analysis of hyponatraemic patients who died in a teaching hospital
}

\section{P. Tzoulis, PM Bouloux \\ Centre for Neuroendocrinology, Royal Free Hospital, London, UK}

\section{Introduction}

Hyponatraemia is associated with increased inpatient mortality, but there is debate about whether hyponatraemia per se contributes to mortality or is merely an epiphenomenon of severe illness.

The objectives of this retrospective chart review were to determine the causes of death and the clinical course of hyponatraemic patients who died during their hospitalisation.

\section{Methods}

A comprehensive retrospective review of case notes, death certificates and laboratory results was conducted for all fatal cases among all inpatients with serum $\mathrm{Na} \leq 128$ $\mathrm{mmol} / \mathrm{l}$ during their hospital stay at Royal Free Hospital between $1^{\text {st }}$ March 2013 and 31 $31^{\text {st }}$ May 2013.

\section{Results}

\begin{tabular}{|lc|}
\hline & Fatal cases \\
& $\mathrm{N}=24$ \\
\hline Age (years) & $75.5(60-83)$ \\
Gender (male/female) & $13: 11$ \\
Admission $\mathrm{Na}(\mathrm{mmol} / \mathrm{l})$ & $132(127-136)$ \\
Lowest $\mathrm{Na}(\mathrm{mmol} / \mathrm{l})$ & $124(122-127)$ \\
SNa at death & $135.5(128-142)$ \\
Exposure to sNa $\leq 128$ & 4 days \\
ICU admission & $6(25 \%)$ \\
ICU LOS (days) & $13.5(5-19)$ \\
LOS (days) & $21.5(10-30)$ \\
\hline
\end{tabular}

\section{Results}

\begin{tabular}{|lc|}
\hline & \\
\hline sNa at time of death & Cases \\
$(\mathrm{mmol} / \mathrm{l})$ & $\mathrm{N}=24$ \\
\hline$<125$ & $2(8.4 \%)$ \\
$126-128$ & $5(20.8 \%)$ \\
$129-134$ & $3(12.5 \%)$ \\
$135-145$ & $11(45.8 \%)$ \\
$>145$ & $3(12.5 \%)$ \\
\hline
\end{tabular}

Among 139 hyponatraemic patients, 24 patients (17.3\%) died during hospitalisation.

9 patients had hypovolaemic hyponatraemia, 8 had SIADH, 3 had hypervolaemic hyponatraemia, and 4 had hyponatraemia of unknown type.

The primary causes of death were: pneumonia (6 cases), malignancy ( 5 cases), cirrhosis ( 4 case), heart failure ( 3 cases), visceral perforation (2 cases), myocardial infarction ( 1 case), acute kidney injury (1 case), stroke (1 case), and cerebral vasculitis (1 case).

The case notes review did not identify any cases of direct contribution of hyponatraemia to death.

\section{Conclusions}

Most hyponatraemic patients who die have severe progressive illnesses.

Hyponatraemia causes death through its severe complications only in rare cases.

Hyponatraemia may contribute indirectly to excess mortality through unknown mechanisms, but physiological studies are needed to examine plausible mechanisms such as possible deleterious effects of hyponatraemia on the immune system and the heart. 